\title{
Simple Harmonic Error Cancellation in Time of Flight Range Imaging
}

\author{
Lee Streeter $^{1 *}$ And Adrian A. Dorrington ${ }^{1}$ \\ ${ }^{1}$ School of Engineering, University of Waikato, Hamilton, New Zealand. \\ *Corresponding author: Ivs2@phys.waikato.ac.nz
}

Compiled October 21, 2015

\begin{abstract}
Amplitude modulated continuous wave time of flight range imaging provides a full field of distance measurement, but common hardware is implemented with digital technology which leads to unwanted harmonic content, a principle source of error in the distance measurements. Existing strategies for correction of harmonics require auxiliary measurements and amplify noise. A small modification of the data acquisition procedure is described which, intrinsically, is invariant to at least one harmonic. The third harmonic, the main cause of harmonic error, is targeted. Compared to traditional measurements the third harmonic is eliminated with no significant increase in noise variance observed. (๑) 2015 Optical Society of America
\end{abstract}

OCIS codes: (110.6880) Three-dimensional image acquistion; (120.3940) Metrology; (280.3640) Lidar; (120.2920) Homodyning

http://dx.doi.org/10.1364/ao.XX.XXXXXX

Homodyne amplitude modulated continuous wave (AMCW) lidar is an active distance measurement technique based on the time of flight (ToF) principle of light. An AMCW camera performs lidar at each pixel, measuring a full image of distances. The advantages of AMCW ToF are fast acquisition of distance images from a single pixel array without the need for sophisticated solutions to inverse problems.

There are known systematic errors in AMCW, one of the most important being harmonic content arising from the square wave modulation in the active imaging process, leading to systematic error in the distance measurements [1-5]. Known methods to correct or reduce harmonic error are (1) to measure the error directly and subtract it [2], (2) to carefully select the number of measurements (a discussion pertaining to the related technique of heterodyne AMCW is given by [1] which is also pertinent to the present work), and (3) to use specific combinations of integration times and phases so that unwanted harmonic content is cancelled (harmonic cancellation) [4]. All these methods suffer from some technical disadvantage, such as an increase in random noise or more involved data acquisition processes than the standard procedure. More recently [6] used multi-frequency techniques to model and correct harmonics error as an alterna- tive to the costly and time consuming process of per-camera measure and substract calibration. Careful selection and fine control of the of the phases has also been proposed for the cancellation of unwanted harmonics [7].

Of the harmonic removal methods harmonic cancellation is best because it does not rely on auxiliary measurement and is independent of instrumental variations such as the modulation bandwidth variation with temperature drift of high speed laser diodes [8]. We report an alternative approach to describing the AMCW demodulation process which leads to a simpler harmonic cancellation method than previously reported [4, 7] that only requires coarse control of the phases, does not requre adjustment of the phase during the capture period, and can be implemented with only minor modifications to traditional hardware.

The homodyne AMCW measurement procedure involves modulation of the light source and sensor at the same rate as each other, $f_{m}$. For ease of implementation, typical hardware is based on digital electronics so use square wave modulation. The light source and sensor are both modulated with a programmable phase offset between them. The sensor measures light backscattered from the scene that travels a distance over a period of time dependent on the speed of light in the medium. This travel time, or ToF of the light, induces a phase shift in the rise and fall times of the light modulation which is encoded as the relative phase offset between the light and the sensor. The integrated intensity is proportional to the overlap in the modulation waveforms of the light source and sensor, and the overlap is in turn dependent on the relative phase offset.

We first consider the AMCW ToF process in the ideal case without harmonic error, and second return below to the implication and correction of square wave harmonics. In the data acquisition a set of $N$ frames are measured, where for each frame we set a distinct phase offset: $N$ evenly distributed phase steps between 0 and $2 \pi$. The measurements, at each pixel, are discrete samples of the correlation between the light source and the sensor waveforms. In the ideal case we assume the waveforms are sinusoidal and the ideal measurements, enumerated $I_{n}$, are

$$
I_{n}=\frac{A}{2} \cos \left(\phi-\frac{2 \pi n}{N}\right)+B, n \in\{0, \ldots, N\},
$$

where $A$ is due to the backscattered light brightness, $B$ is an additive term with contributions from ambient light and the light source, and $\phi$ is the phase offset between the sensor and the 
light return induced by the light travel distance. The relationship between phase $\phi$ and distance $d$ is

$$
\phi=\frac{4 \pi d f_{m}}{c}
$$

where $c$ is the speed of light in the medium. Typically $N=4$, and recalling Euler's formula, the demodulation (computation of the complex range phasor estimate, $p$ ) is

$$
p=A e^{i \phi}=\left(I_{0}-I_{2}\right)+i\left(I_{1}-I_{3}\right),
$$

where $i$ is the imaginary unit. The phase estimate $\phi$ is the angle of $p$, recovered using the usual trigonometric operation

$$
\phi=\tan ^{-1}\left(\frac{\Im p}{\Re p}\right) \text {. }
$$

It is a simple matter to reconstruct $p$ from the $I_{n}$ via the following alternative but equivalent steps. Compute the complex measurements,

$$
\begin{aligned}
& m_{0}=\frac{p}{2}+C=I_{0}+i I_{1} \\
& m_{2}=-\frac{p}{2}+C=I_{2}+i I_{3}
\end{aligned}
$$

where $C=B+i B$ is a complex constant due to ambient light. In a more general form we have

$$
m_{\alpha}=\frac{p i^{\alpha}}{2}+C, \quad \alpha \in\{0,2\} .
$$

Recovery of $p$ is straightforward:

$$
p=2 \frac{m_{2}-m_{0}}{i^{2}-1}
$$

We now return to the case of harmonic error due to digital square wave modulation. Square wave modulation introduces odd order harmonicss to the waveform. Assuming the light source and sensor are modulated by a square wave function with $50 \%$ duty cycle, the correlation waveform is (cf Eqn. 1)

$$
I_{\alpha}=B+\frac{A}{2} \sum_{\kappa=1,3,5, \ldots}^{\infty} \frac{1}{\kappa^{2}} \cos \left(\kappa \phi+\kappa \frac{\pi}{2} \alpha\right)
$$

where $\alpha$ is a phase offset coefficient distinct from $n$. Let $p$ be the true range phasor without unwanted harmonic content, as above, and let $\hat{p}$ below be the estimate with contamination by the unwanted harmonics. The result of the correlation of the square wave source and sensor functions is a triangle wave which produces the following mathematical form in the complex measurements

$$
\begin{aligned}
& m_{\alpha}=I_{\alpha}+i I_{\alpha+1} \\
& \quad=C+p_{1} i^{\alpha}+p_{-3} i^{-3 \alpha} \frac{1}{3^{2}}+p_{5} i^{5 \alpha} \frac{1}{5^{2}}+\ldots,
\end{aligned}
$$

where $p_{\kappa}=A e^{i \kappa \phi} / 2$. Let one value for $\alpha$ be 0 , leave the second to be determined, and form the estimate for the range phasor from the following generalisation of Eqn. 8

$$
\hat{p}_{\alpha}=2 \frac{m_{\alpha}-m_{0}}{i^{\alpha}-1} .
$$

Substitution of Eqn. 10 into Eqn. 11 gives

$$
\hat{p}_{\alpha}=p_{1}+p_{-3} \frac{i^{-3 \alpha}-1}{i^{\alpha}-1} \frac{1}{3^{2}}+p_{5} \frac{i^{5 \alpha}-1}{i^{\alpha}-1} \frac{1}{5^{2}}+\cdots .
$$

Immediately we see that when

$$
i^{3 \alpha}=1 \Rightarrow \alpha=\frac{4 j}{3}, j \in\{1,2,3, \ldots\},
$$

then the third harmonic is cancelled. This harmonic cancellation is achieved by relaxing the constraint that the phase steps are evenly spaced. This result is a considerable improvement for such a small change to traditional four phase step AMCW ToF. Note that $j=3$ is equivalent to $j=0$ and the coefficients proceed to cycle. A pseudo-code example of AMCW with cancellation of the third harmonic is given in Alg. 1 for $\alpha=4 / 3$. A total of four raw frames are acquired from which $\hat{p}_{4 / 3}$ is computed with the third harmonic cancelled.

Algorithm 1. Third harmonic cancellation AMCW.

$$
\begin{aligned}
& \text { 1: } \text { for all } \alpha \in\{0,4 / 3\} \text { do } \\
& \text { 2: } \quad \text { Acquire } I_{\alpha} \\
& \text { 3: } \quad \text { Acquire } I_{\alpha+1} \\
& \text { 4: } \quad m_{\alpha} \leftarrow I_{\alpha}+i I_{\alpha+1} \\
& \text { 5: } \hat{p}_{4 / 3} \leftarrow 2 \frac{m_{4 / 3}-m_{0}}{i^{4 / 3}-1}
\end{aligned}
$$

Removal of higher order harmonics follows naturally by taking further measurements. To describe higher order cancellation we describe cancellation of the fifth harmonic, cancellation of further harmonics follows by induction. First note the following explicit result of the cancellation of the third harmonic with $j=1$,

$$
\hat{p}_{\frac{4}{3}}=p_{1}+p_{5} \frac{e^{\frac{i \pi}{3}}}{5^{2}}+\cdots .
$$

Repetition of the third harmonic cancellation with an extra phase shift according to coefficient $\beta$ gives

$$
\hat{p}_{\frac{4}{3}, \beta}=p_{1} i^{\beta}+p_{5} i^{5 \beta} \frac{e^{\frac{i \pi}{3}}}{5^{2}}+\cdots .
$$

Therefore, from Eqns. 14-15 and Eqn. 12, we deduce that $\beta=4 / 5$ corresponds to cancellation of the fifth harmonic. Furthermore, we can divide the acquisition period and accumulate the measured data in digital memory [4]. Such combinations of techniques may be employed ad infinitum to beneficial effect.

We experimentally test the rejection of the third harmonic and compare to traditional four even phase step AMCW range measurement. A proprietary prototype ToF camera is used. The camera is arranged to image a target of matte white foam board which has high reflectance. A region of interest of one hundred and twenty pixels on the image of the target is carefully segmented, to choose data of adequate signal quality while avoiding saturated pixels, and averaged before analysis.

The camera is operated with a modulation frequency of $70 \mathrm{MHz}$. The phase step domain must be divided into a multiple of twelve even phase steps so that both harmonic cancellation and traditional operation can be performed with the same configuration, ensuring fairness in the comparison of the two methods. The phase step domain is divided in to $N=36$ evenly spaced positions. Under this regime, traditional AMCW corresponds to measurements at phase steps $n \in\{0,9,18,27\}$. The third order harmonic cancellation uses positions $n \in\{0,9\}$ for $\alpha=0$ and $n \in\{12,21\}$ for $\alpha=4 / 3$.

The phase offset between the light source and the sensor is incremented over the thirty six steps in hardware, producing an artificial phase sweep from which the harmonics are examined. 
One hundred and twenty range images are taken at each of the thirty six phase step increments. To compare between the acquisition methods we: perform direct examination of the distance measurement error; Fourier analysis to examine the negative third harmonic; and examine the standard deviation (STD) in the phase.

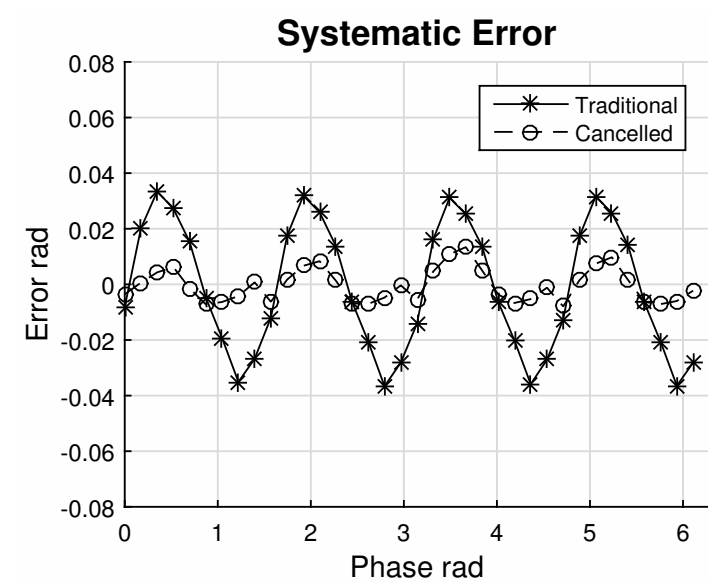

Fig. 1. Mean phase error.

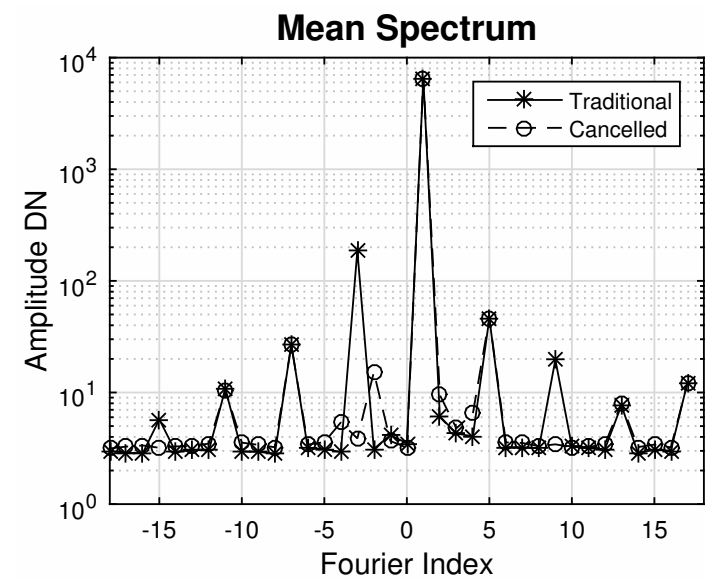

Fig. 2. Fourier spectrum for $\alpha=4 / 3$ compared to even phase steps. The fundamental is the largest peak, the third harmonics are the peaks either side of the fundamental.

The phase linearity shows a marked improvement, see Fig. 1. The traditional even phase step AMCW we see the familiar four cycle error due to the third and fifth harmonics. The harmonic cancellation corrected error is dominated by the much smaller four cycle error due to the remaining fifth harmonic. The peak to peak phase error is reduced from $0.07 \mathrm{rad}$ to $0.02 \mathrm{rad}$ (precision is given in Fig. 3 below).

From Fourier analysis of the complex range phasors, Fig. 2, the mean (STD) of amplitude of the negative third harmonic before correction is 186 (3) DN, and with correction 4(2) DN. A two sample t-test shows that we can reject the hypothesis that the mean spectral feature at the negative third bins are equal $(H=1$, $p \ll 0.01$ ), which supports the claim that the cancellation of the negative third harmonic is successful. Let $k$ be the Fourier index. There is an increase in the spectral content visible at $k=-2$ and $k=-4$, the cause of which is unknown, but is believed to be a subtlety of the camera hardware and not an artefact of the

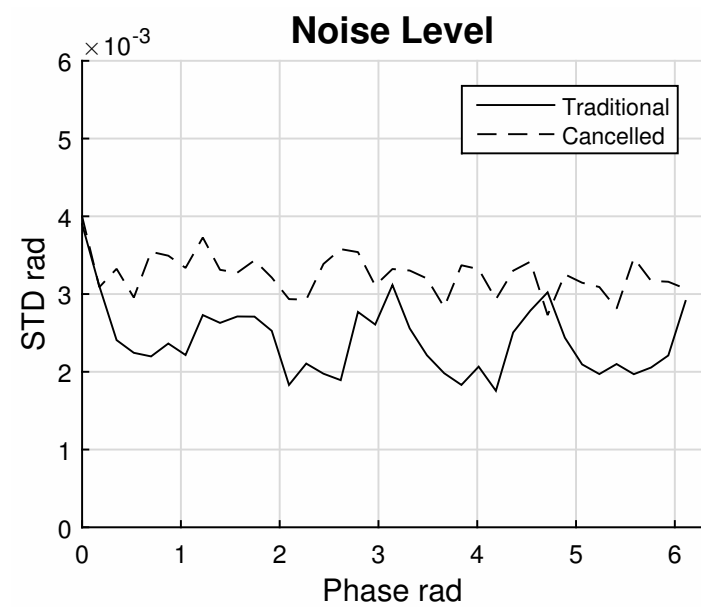

Fig. 3. Standard deviation in phase for $\alpha=4 / 3$. The data cover a phase sweep over one complete ambiguity distance.

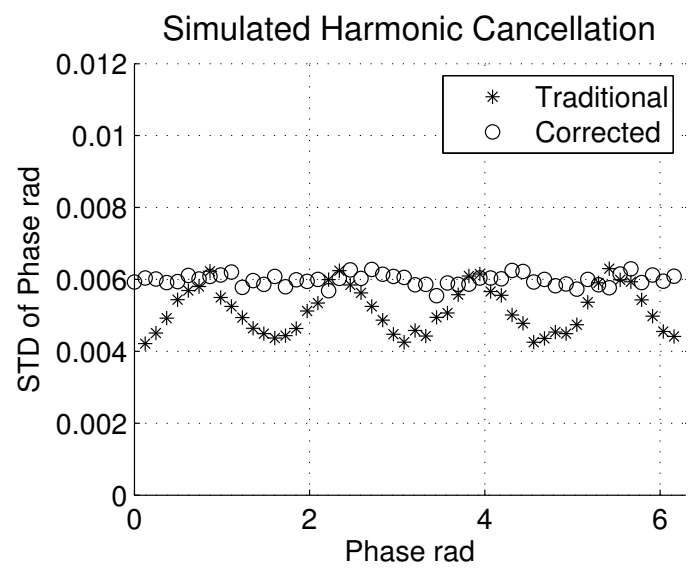

Fig. 4. Simulation of AMCW ToF without jitter, both traditional four phase step and with cancellation of the third harmonic.

harmonic cancellation. A decisive explanation is left for future work. The ninth and fifteenth harmonics, $k=\{9,-15\}$, being multiples of three, are also removed by the cancellation process that targets the third.

The STD with distance, Fig. 3 indicates that the harmonic cancellation reduces the dependence on phase of precision that is seen in traditional four phase step AMCW ToF. Visually we observe a small increase in noise (reduction of precision). However, a F-test over all phase estimates in the data shows that the null hypothesis, that the difference in variance of the phase between the traditional method and the proposed harmonic cancellation is zero, cannot be rejected $(H=0, p=0.96)$, which supports the assertion that the harmonic cancellation has no significant effect on the random noise.

Simulations are performed to gain further insight into the effect of the proposed harmonic cancellation on noise. Additive Gaussian noise and Gaussian jitter are both investigated. The modelling of jitter is motivated by recent results $[9,10]$ that demonstrate that it is an important noise source in AMCW ToF, and to test if jitter has an affect on noise variance with respect to the phase. In [9] the effect of jitter is not reduced by increased backscattered light power, but in [10] it is reduced. This discrep- 


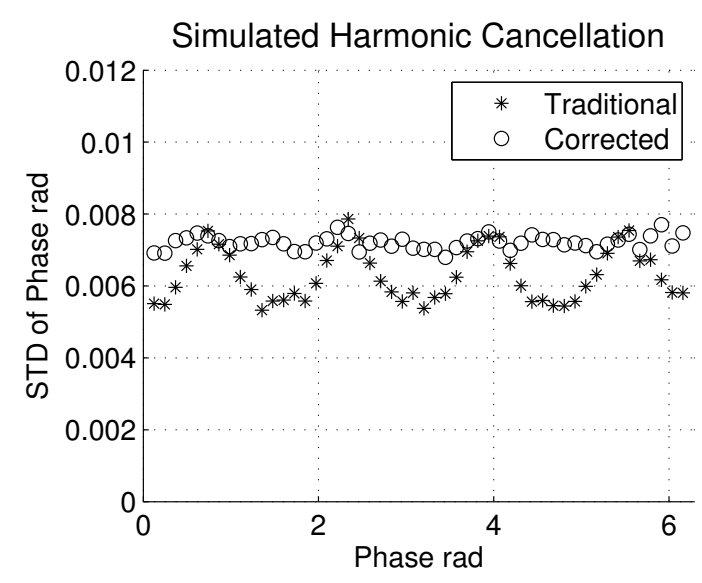

Fig. 5. Simulation of AMCW ToF with jitter, both traditional four phase step and with cancellation of the third harmonic.

ancy is resolved by understanding that the effect of jitter noise is not reduced by increased backscattered light power alone but, assuming zero mean random fluctuations, is reduced by the averaging process induced by integration over a finite time period.

Let $\theta_{n}$ be the phase offset for image frame $I_{n}$, additive noise $\epsilon_{n} \sim \mathcal{N}(0, \sigma)$, jitter noise $\epsilon_{j, n} \sim \mathcal{N}\left(0, \sigma_{j}\right)$, and $A_{P} \sim \mathcal{P}(A)$ be a Poisson random variable with parameter $A$ representing the random fluctuations in the light. The noise is modelled as

$$
I_{n}=A_{P} \sum_{l=1,3,5, \ldots}^{M} \frac{1}{l^{2}} \cos \left(l\left(\phi+\theta_{n}+\epsilon_{j, n}\right)\right)+B+\epsilon_{n},
$$

and the simulations proceed using the same phase steps (values of $\theta_{n}$ ) as the measurements above. Two simulations are performed, one without jitter and the other modelling jitter. In both the simulations $A=10^{5}$, the additive noise level is $\sigma=10^{3}$. In the simulation with jitter $\sigma_{\mathrm{j}}=1 / 45$. The third and fifth harmonics are modelled. The simulations are repeated one-thousand times, and the standard deviation is computed for each phase $\phi$.

We present the noise results for the simulation without jitter in Fig. 4, and with jitter in Fig. 5. The shape of both simulations visually resemble the result in Fig. 3 up to a circular shift in phase. The inclusion of jitter has no apparent affect on the shape of the variance with respect to phase, but instead increases the overall variance evenly. The corrected noise level is at least as large as the traditional AMCW noise at all phases. A reasonable explanation is that unwanted harmonics add to the total signal power, which in turn increases the SNR, but at the cost of biasing error. Overall the simulations indicate that it is the harmonics, and not jitter, that dominates the shape of the variance with respect to phase.

In summary we have demonstrated harmonic cancellation in AMCW ToF four phase step measurement with only a small change to the acquisition process. The third harmonic was successfully removed. No significant change in standard deviation due to harmonic cancellation was detected. The method is easily extensible to higher order harmonics.

\section{ACKNOWLEDGEMENTS}

We acknowledge Michael J. Cree for his useful discussion.

\section{REFERENCES}

1. A. A. Dorrington, M. J. Cree, D. A. Carnegie, and A. D. Payne, "Selecting signal frequencies for best performance of fourier-based phase detection," in "Proceedings of the Twelfth New Zealand Electronics Conference," (Manukau City, New Zealand, 2005), pp. 189 - 193.

2. M. Lindner and A. Kolb, "Lateral and depth calibration of PMD-distance sensors," in "Advances in Visual Computing, Part II," , vol. 4292 (Springer-Verlag, 2006), vol. 4292, pp. 524-533.

3. M. Lindner, A. Kolb, and T. Ringbeck, "New insights into the calibration of tof sensors," in "IEEE Conf. on Computer Vision and Pattern Recognition (CVPR), Workshop on ToF Camera based Computer Vision (TOFCV)," (IEEE, 2008), pp. 1-5. DOI 10.1109/CVPRW.2008.4563172.

4. A. D. Payne, A. A. Dorrington, M. J. Cree, and D. A. Carnegie, "Improved measurement linearity and precision for amcw time-of-flight range imaging cameras," Applied Optics 49 (2010).

5. J. P. Godbaz, M. J. Cree, and A. A. Dorrington, "Understanding and ameliorating non-linear phase and amplitude responses in amcw lidar," Remote Sensing 4, 21-42 (2012).

6. M. Feigin, R. Whyte, A. Bhandari, A. Dorington, and R. Raskar, "Modeling wiggling as a multi-path interference problem in AMCW ToF imaging," OPTICS EXPRESS 23, 19213-19225 (2015).

7. C. Peters, J. Klein, M. B. Hullin, and R. Klein, "Solving trigonometric moment problems for fast transient imaging," ACM Transactions on Graphics (Proc. SIGGRAPH Asia) 34, in press (2015).

8. G. Keiser, Optical Fiber Communications (McGraw-Hill, Inc, New York, NY, USA, 1991).

9. L. Streeter, M. J. Cree, and A. A. Dorrington, "A strategy for the correction of effects of jitter in AMCW lidar images," in "The 28th International Conference of Image and Vision Computing New Zealand," (Wellington, New Zealand, 2013), pp. 500-505.

10. J. Seiter, M. Hofbauer, M. Davidovic, and H. Zimmermann, "Investigation of the distance error induced by cycle-to-cycle jitter in a correlating time-of-flight distance measurement system," Optical Engineering 53, 073104 (2014). 


\section{FIFTH INFORMATIONAL PAGE}

\section{REFERENCES}

1. A. A. Dorrington, M. J. Cree, D. A. Carnegie, and A. D. Payne, "Selecting signal frequencies for best performance of fourier-based phase detection," in "Proceedings of the Twelfth New Zealand Electronics Conference," (Manukau City, New Zealand, 2005), pp. 189 - 193.

2. M. Lindner and A. Kolb, "Lateral and depth calibration of PMD-distance sensors," in "Advances in Visual Computing, Part II," , vol. 4292 (Springer-Verlag, 2006), vol. 4292, pp. 524-533.

3. M. Lindner, A. Kolb, and T. Ringbeck, "New insights into the calibration of tof sensors," in "IEEE Conf. on Computer Vision and Pattern Recognition (CVPR), Workshop on ToF Camera based Computer Vision (TOFCV)," (IEEE, 2008), pp. 1-5. DOI 10.1109/CVPRW.2008.4563172.

4. A. D. Payne, A. A. Dorrington, M. J. Cree, and D. A. Carnegie, "Improved measurement linearity and precision for amcw time-of-flight range imaging cameras," Applied Optics 49 (2010).

5. J. P. Godbaz, M. J. Cree, and A. A. Dorrington, "Understanding and ameliorating non-linear phase and amplitude responses in amcw lidar," Remote Sensing 4, 21-42 (2012).

6. M. Feigin, R. Whyte, A. Bhandari, A. Dorington, and R. Raskar, "Modeling wiggling as a multi-path interference problem in AMCW ToF imaging," OPTICS EXPRESS 23, 19213-19225 (2015).

7. C. Peters, J. Klein, M. B. Hullin, and R. Klein, "Solving trigonometric moment problems for fast transient imaging," ACM Transactions on Graphics (Proc. SIGGRAPH Asia) 34, in press (2015).

8. G. Keiser, Optical Fiber Communications (McGraw-Hill, Inc, New York, NY, USA, 1991).

9. L. Streeter, M. J. Cree, and A. A. Dorrington, "A strategy for the correction of effects of jitter in AMCW lidar images," in "The 28th International Conference of Image and Vision Computing New Zealand," (Wellington, New Zealand, 2013), pp. 500-505.

10. J. Seiter, M. Hofbauer, M. Davidovic, and H. Zimmermann, "Investigation of the distance error induced by cycle-to-cycle jitter in a correlating time-of-flight distance measurement system," Optical Engineering 53, 073104 (2014). 